\title{
A rare case of acute promyelocytic leukemia with focal bone marrow involvement presenting as a paraspinal myeloid sarcoma
}

\author{
Meggie E. Doucet ${ }^{1} \cdot$ John J. Schmieg ${ }^{1}$
}

Received: 23 July 2016 / Accepted: 18 January 2017 / Published online: 1 February 2017

(C) Springer-Verlag Berlin Heidelberg 2017

\begin{abstract}
Acute promyelocytic leukemia (APL) accounts for 5-8\% of acute myeloid leukemias (AMLs). Myeloid sarcomas (MS) are rare extramedullary tumors consisting of immature myeloid cells. These tumors may develop de novo or concurrently with AML and may precede or coincide with the occurrence of AML. The occurrence of MS in an APL patient is a rare clinical event. In these cases, it usually occurs as a sign of relapse. Focal bone marrow involvement by APL is also rare; the majority of cases of APL demonstrate a diffusely hypercellular bone marrow throughout the axial skeleton due to expansion of neoplastic blasts/promyelocytes. We describe a 23-year-old female who presented with gait problems, including multiple falling episodes, and lower extremity weakness. An MRI demonstrated a paraspinal soft tissue mass. Histopathologic analysis demonstrated a myeloid sarcoma expressing CD45, myeloperoxidase, CD117, BCL2, focal weak CD34, and weak CD99 and negative for CD30, TdT, CD20, CD3, and CD10. The bone marrow aspirate demonstrated blasts/promyelocytes accounting for approximately $26 \%$ of the total bone marrow aspirate cellularity. The bone marrow biopsy and clot section demonstrated normocellularity with a few blast/promyelocyte foci on the clot section accounting for approximately 5\% of total bone marrow cellularity. These foci expressed MPO and CD117 and were negative for CD34. Flow cytometry demonstrated
\end{abstract}

Meggie E. Doucet

mdoucet3@tulane.edu

John J. Schmieg

jschmieg@tulane.edu

1 Department of Pathology and Laboratory Medicine, Tulane University School of Medicine, 1430 Tulane Ave, New Orleans, LA 70112, USA blasts accounting for approximately $31 \%$ of all CD45+ events expressing MPO, CD33, CD13, CD117, CD64, CD2, CD38, and CD71 and negative for CD34, HLA-DR, CD11b, CD14, CD15, CD16, CD19, CD22, CD10, CD56, CD61, and surface and cytoplasmic CD3. Fluorescence in situ hybridization (FISH) analysis was positive for the $\mathrm{t}(15 ; 17)(\mathrm{q} 22 ; \mathrm{q} 12)$ promyelocytic leukemia/retinoic acid receptor alpha (PML/ RARA) translocation. The patient was induced with arsenic trioxide and subsequently underwent consolidation chemotherapy with daunorubicin and all-trans-retinoic acid. Her postchemotherapy bone marrow biopsy was negative for residual disease. Flow cytometry and FISH analysis were also negative. This is a rare case of APL involving both the initial presentation as myeloid sarcoma and focal bone marrow involvement.

Keywords Acute promyelocytic leukemia $\cdot$ Myeloid sarcoma $\cdot$ Paraspinal $\cdot$ Focal bone marrow involvement

\section{Introduction}

Acute promyelocytic leukemia (APL) is defined by the World Health Organization Classification of Tumors of Hematopoietic and Lymphoid Tissues as an acute myeloid leukemia (AML) in which abnormal promyelocytes predominate and is defined by the $\mathrm{t}(15 ; 17)(\mathrm{q} 22 ; \mathrm{q} 12)$ translocation. APL accounts for $5-8 \%$ of acute myeloid leukemias. The disease occurs predominantly in middle-aged adults but can occur at any age. Typically, the bone marrow of patients with APL is diffusely hypercellular throughout the axial skeleton consisting mostly of neoplastic blasts/promyelocytes [1].

Myeloid sarcomas (MS) are rare tumors that are comprised of myeloid blasts with or without maturation occurring at an anatomical site other than the bone marrow. Almost every site of the body can be involved, in particular the skin, lymph 
node, gastrointestinal tract, bone, soft tissue, and testis. These tumors may develop de novo or concurrently with AML and may precede or coincide with the occurrence of AML. MS may also be the initial manifestation of relapse in a patient with previously diagnosed AML regardless of blood or bone marrow findings. MS associated with APL is an extremely rare event and in most cases occurs at the time of relapse [2]. In patients with APL, extramedullary disease most commonly occurs in the central nervous system (CNS). Greater than $10 \%$ of hematologic relapses are accompanied by CNS involvement. We present a rare case of APL involving both the initial presentation as myeloid sarcoma and focal bone marrow involvement.

\section{Case presentation}

The patient is a previously healthy 23 -year-old AfricanAmerican female with no past medical history, who was in her usual state of health until 3 weeks before presentation when she started to experience gait problems, including multiple falling episodes, and lower extremity weakness. At presentation, her physical examination demonstrated decreased sensation below the breast line and decreased bilateral lower extremity motor power (4/5). The remainder of her exam was unremarkable. A CBC demonstrated a hemoglobin level of $9.1 \mathrm{~g} / \mathrm{dL}$, a hematocrit of $27.4 \%$, a white blood cell count of $8800 / \mathrm{mm}^{3}$, and a platelet count of $117,000 / \mathrm{mm}^{3}$. A subsequent MRI demonstrated a large circumferential epidural paraspinal soft tissue mass spanning from $\mathrm{T} 3$ to $\mathrm{T} 5$ measuring approximately $6 \mathrm{~cm}$ in the greatest dimension with significant spinal cord compression.

She underwent a CT-guided biopsy that demonstrated a diffuse proliferation of medium-to-large-sized mononuclear cells with irregular indented reniform nuclei, a moderate amount of granular eosinophilic cytoplasm, finely dispersed chromatin, and occasional prominent nucleoli consistent with immature myeloid cells (Fig. 1a). Immunostaining for CD45, myeloperoxidase, and CD117 demonstrated diffuse positivity in the tumor cells (Fig. 1b-d) whereas staining for CD34 demonstrated focal weak positivity (Fig. 1f). The tumor cells also showed diffuse positivity for BCL2 (Fig. 1e) and vimentin and weak positivity for CD99. The tumor cells were negative for CD30, TdT, CD20, CD3, CD10, CD5, AE1/AE3, Cam 5.2, synaptophysin, desmin, and myogenin. Based on these findings, the mass was diagnosed as an extramedullary myeloid tumor (myeloid sarcoma). A subsequent excisional biopsy with decompression laminectomy of T3 to T5 was performed in order to relieve the cord compression and treat her neurologic symptoms. Unfortunately, the patient became paraplegic after the procedure with absent bilateral lower extremity motor power $(0 / 5)$, absent bilateral lower extremity reflexes $(0 / 2)$, and complete absence of sensation below the level of T5 bilaterally.

A bone marrow biopsy and aspirate were subsequently performed. The bone marrow aspirate demonstrated atypical blast forms accounting for approximately $26 \%$ of the total aspirate cellularity. Most of the blasts demonstrated a moderate amount of basophilic cytoplasm containing numerous azurophilic granules with perinuclear hofs (Fig. 2f). In addition, the nuclei were predominately round in shape with open chromatin and occasional prominent nucleoli. No definitive Auer rods were seen. The bone marrow biopsy and clot section were normocellular for age with a few blast/promyelocyte foci seen on the clot section accounting for approximately $5 \%$ of total bone marrow cellularity (Fig. 2a, b). These foci expressed myeloperoxidase (Fig. 2c) and CD117 (Fig. 2d) and were negative for CD34 (Fig. 2e). Flow cytometry (Fig. 3) demonstrated blasts accounting for approximately $31 \%$ of all CD $45+$ events expressing myeloperoxidase, CD33, CD13, CD117, CD64, CD2, and CD38 and negative for CD34, HLA-DR, CD11b, CD14, CD15, CD16, CD19, CD22, CD10, CD56, CD61, and surface and cytoplasmic CD3. Fluorescence in situ hybridization (FISH) analysis (Fig. 4) was positive for the $\mathrm{t}(15 ; 17)(\mathrm{q} 22 ; \mathrm{q} 12)$ promyelocytic leukemia/retinoic acid receptor alpha (PML/RARA) translocation; there was no evidence of $\mathrm{t}(8 ; 21)(\mathrm{q} 22 ; \mathrm{q} 22)$ or any other specific cytogenetic abnormalities.

The patient was induced with arsenic trioxide and subsequently underwent consolidation chemotherapy with daunorubicin and all-trans-retinoic acid. Her post-chemotherapy bone marrow biopsy was negative for residual disease. Flow cytometry and FISH analysis were also negative. She is currently receiving maintenance therapy with methotrexate/6-mercaptopurine/all-trans-retinoic acid (ATRA) and is disease free.

\section{Discussion}

APL is an acute myeloid leukemia in which abnormal promyelocytes predominate in the bone marrow and/or the peripheral blood. APL comprises 5-8\% of all AMLs. It can occur at any age but is predominantly seen in middle-aged adults. There are two subtypes of APL, the classical hypergranular variant and the microgranular variant. Patients with either subtype can present with disseminated intravascular coagulation. In patients with the microgranular variant, the peripheral blood leukocyte count can be very high with a rapid doubling time. This is typically not seen in the classical variant of APL [1]. MS associated with APL is an extremely rare event and in most cases occurs at the time of relapse [2]. In patients with APL, extramedullary disease most commonly occurs in the central nervous system (CNS) with greater than $10 \%$ of hematologic relapses accompanied by CNS involvement. 
Fig. 1 Paraspinal mass. The paraspinal mass was composed of medium to large cells with irregular indented or reniform nuclei and moderate amounts of cytoplasm with occasional prominent nucleoli (a H\&E stain, $400 \times)$. Tumor cells were positive for CD45 (b CD45 stain, 400×), myeloperoxidase (c MPO stain, $400 \times$ ), CD117 (d CD117 stain, 400×), and BCL2 (e BCL2 stain, $400 \times)$ and were focally weakly positive for CD34 (f CD34 stain, $400 \times)$
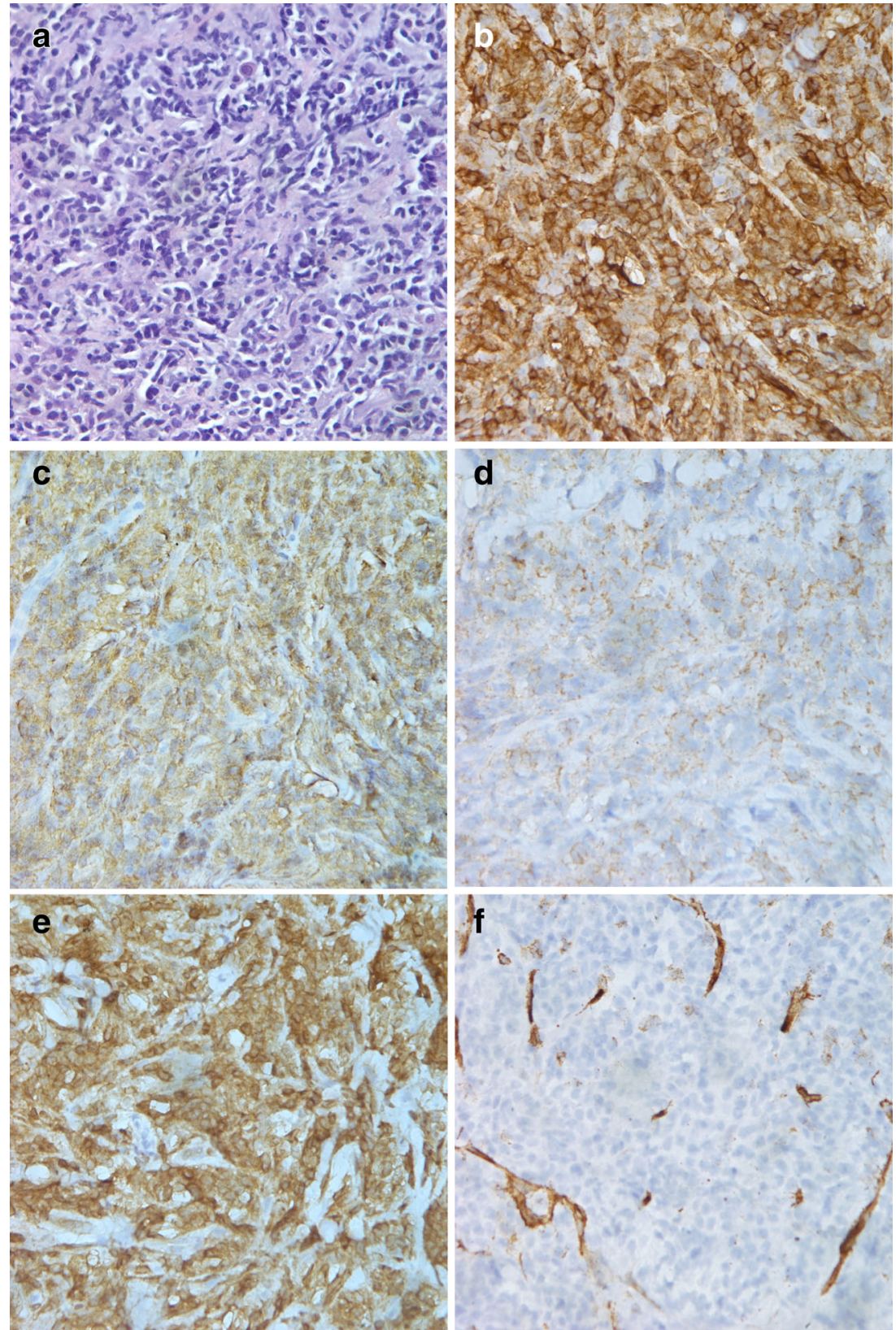

Morphologically, the neoplastic promyelocytes of the classical hypergranular variant of APL typically show kidneyshaped or bilobed nuclei demonstrating finely dispersed chromatin and occasional prominent nucleoli. The cytoplasm of the classical hypergranular variant of APL characteristically contains numerous densely-packed to coalescent primary-type granules that stain bright pink, red, or purple on Romanowsky stains. Auer rods, whether singly or in compact bundles, are a common finding. In the microgranular variant of APL, the leukemic cells demonstrate a paucity or absence of granules on light microscopy with predominantly bilobed nuclei exhibiting finely dispersed chromatin and occasional prominent nucleoli. Despite the lack of significant granularity on light microscopy in microgranular APL, ultrastructural studies reveal the presence of numerous small granules (microgranules) that fail to stain due to their small size. As can be seen in the classical hypergranular variant of APL, occasional cells with Auer rods can be seen in microgranular variant APL as well. The bone marrow biopsy of APL regardless of subtype is typically hypercellular with little to no residual background trilineage hematopoiesis. Immunophenotypically, the atypical promyelocytes are characterized by low expression or absence of HLA-DR, CD34, CD11a, CD11b, and CD18. Most cases show bright expression of CD33, dim expression of CD117, and moderate expression of CD64. CD15 and CD65 are usually negative or at most weakly positive. Expression of CD56, 
Fig. 2 Bone marrow clot. The bone marrow demonstrated focal involvement with few blast/ promyelocyte foci on the clot section accounting for approximately $5 \%$ of total bone marrow cellularity (a H\&E stain, 100×; b H\&E stain, 400×). These foci expressed myeloperoxidase (c MPO stain, 200×) and CD117 (d CD117 stain, 200×) and were negative for CD34 (e CD34 stain, $200 \times$ ). The blasts demonstrated abundant basophilic cytoplasm, some with numerous azurophilic granules and perinuclear hofs (f Wright-Giemsa stain, 1000×)

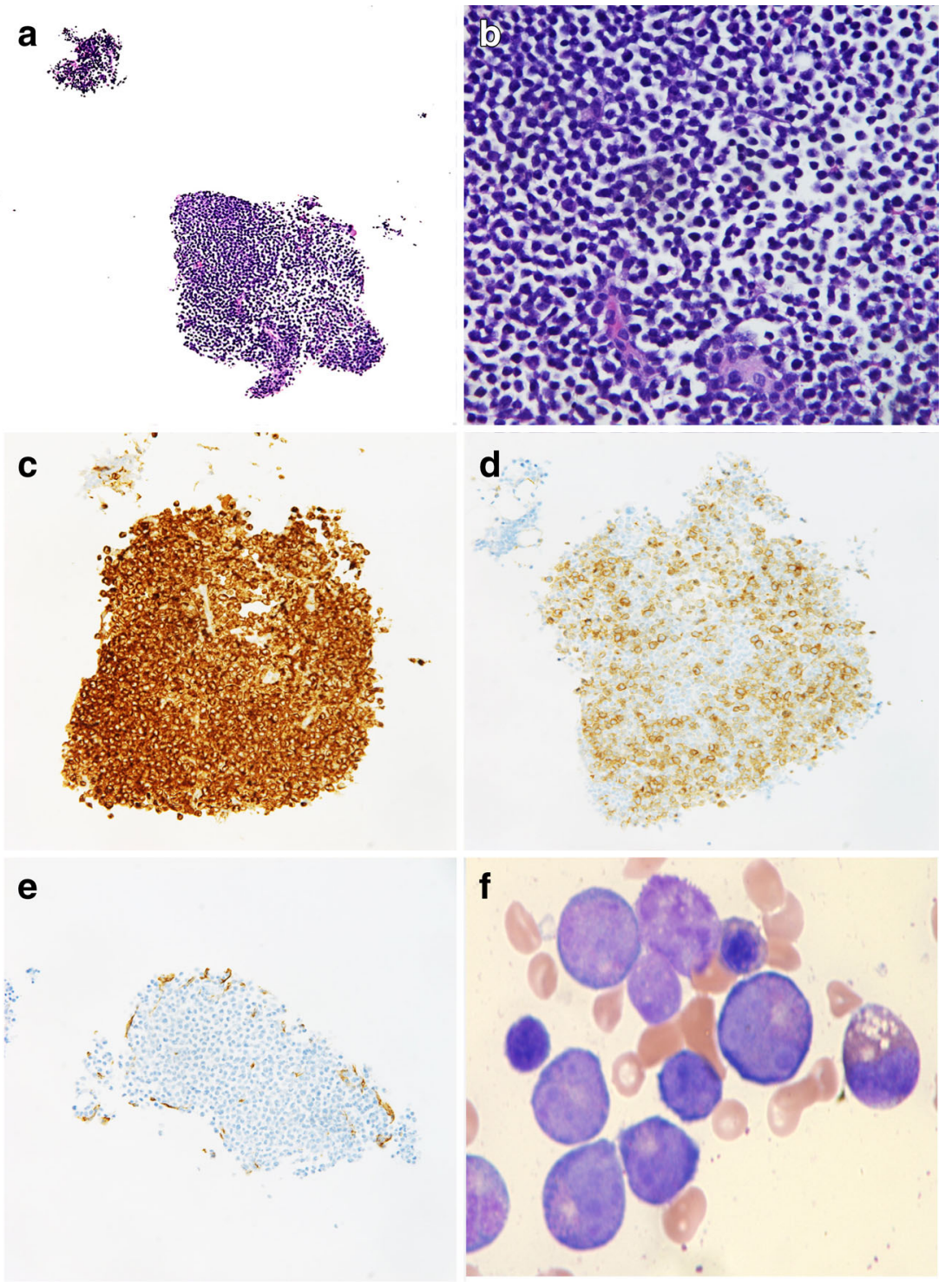

which is seen in approximately $20 \%$ of APL patients, is associated with a worse outcome. The classic cytogenetic finding of APL regardless of subtype is the fusion of the RARA gene on chromosome 17q12 and the PML nuclear regulatory factor gene on chromosome $15 \mathrm{q} 22$, although variant translocations and secondary cytogenetic abnormalities can be identified [1].

MS at diagnosis or relapse occurs in 3-8\% of AML patients. They occur more frequently in patients with AMLs with myelomonocytic or monocytic morphology and can be associated with a variety of chromosomal abnormalities, including $\mathrm{t}(8 ; 21)(\mathrm{q} 22 ; \mathrm{q} 22)$ translocation $[3,4]$. An interesting finding in our case is that although the blasts showed the typical $\mathrm{t}(15 ; 17)(\mathrm{q} 22 ; \mathrm{q} 12)$ translocation and immunophenotype characteristic of APL, their cytomorphologic features were more in keeping with those typically seen in AML with $\mathrm{t}(8 ; 21)(\mathrm{q} 22 ; \mathrm{q} 22)$, namely, large size, abundant basophilic cytoplasm with numerous azurophilic granules and perinuclear hofs, predominately round nuclei, finely dispersed chromatin, and occasional prominent nucleoli (Fig. 2f). The morphologic similarity of the blasts in this case to those usually seen in AML with $\mathrm{t}(8 ; 21)(\mathrm{q} 22 ; \mathrm{q} 22)$, as well as the clinical presentation of MS, which is more commonly seen with AML with $\mathrm{t}(8 ; 21)(\mathrm{q} 22 ; \mathrm{q} 22)$, underscores the importance of ancillary studies, in particular, cytogenetics and flow cytometry, in arriving at the correct diagnosis.

Focal bone marrow involvement is rarely observed in patients with AML, in particular APL. It is more often described in patients with leukemic relapse after chemotherapy or hematopoietic stem cell transplantation and can be seen in patients with lymphoplasmacytic neoplasms and solid tumors [5, 6]. The mechanism behind focal involvement of AML is unclear. Failure of the graft-versus-leukemia effect in specific extramedullary sites has been postulated in those patients presenting with localized relapse following hematopoietic stem 
a

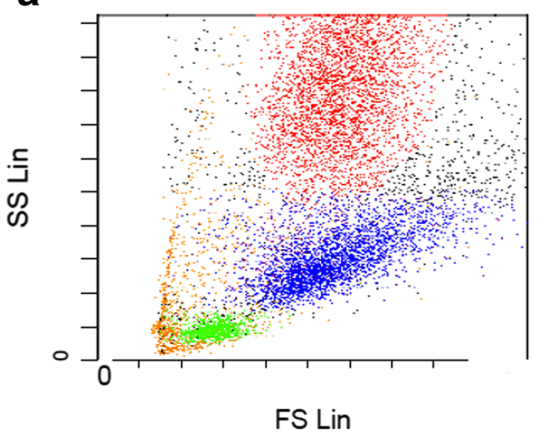

d

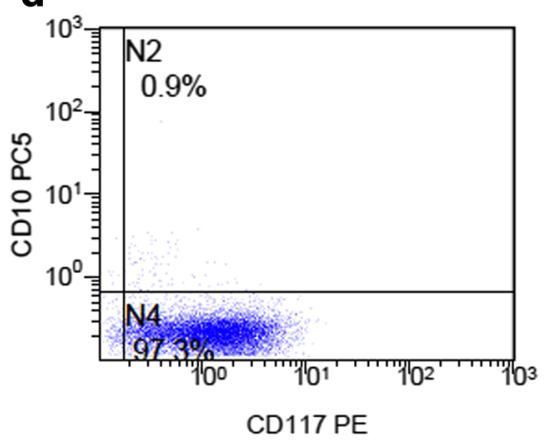

b

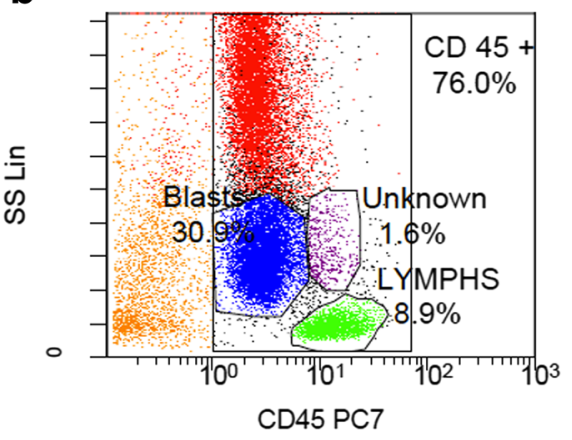

e

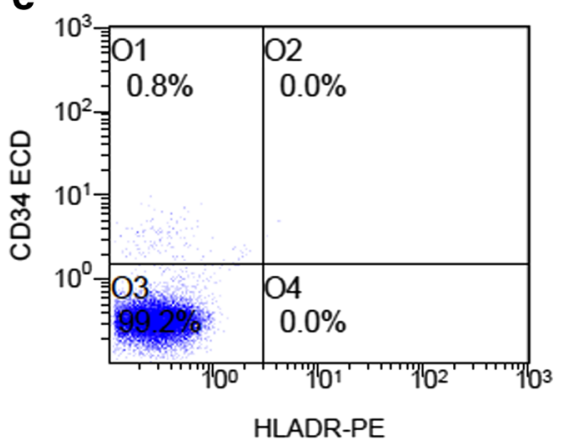

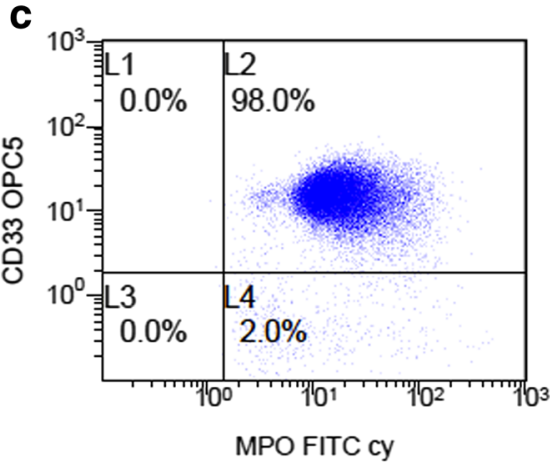

f

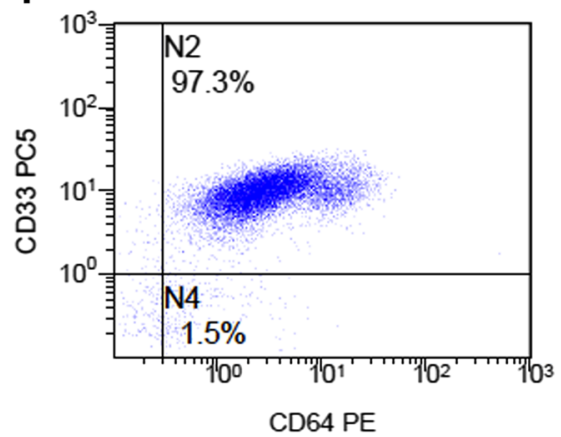

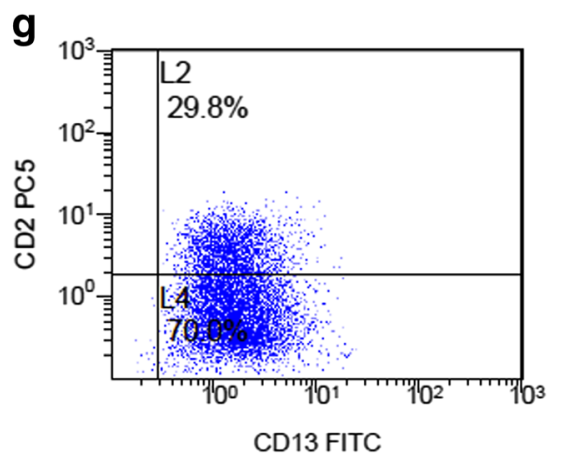

CD13 FITC

Fig. 3 Flow cytometry of bone marrow. The ungated plots demonstrate a population of blasts (blue) comprising approximately $31 \%$ of all events with high forward scatter and intermediate side scatter (a) in addition to dim CD45 expression (b). This population expressed bright MPO (c), bright CD33 (c and f), dim to moderate CD117 (d), partial aberrant

cell transplantation [7]. Heterogeneity in the bone marrow microenvironment is another possible explanation as to why myeloid blasts are able to proliferate in one site over another [6]. Certain patterns of expression of cell-surface adhesion molecules by clonal cells are yet another possible explanation for a particular anatomic distribution [6].

APL is sensitive to treatment with ATRA, which induces the differentiation and maturation of the neoplastic promyelocytes to mature neutrophils, which then undergo apoptosis. The prognosis in patients treated with ATRA and an anthracycline is more favorable than for any other AML cytogenetic subtype. Cases of relapsed or refractory APL show a good response with arsenic trioxide therapy. Expression of CD56 is associated with a less favorable prognosis. In this

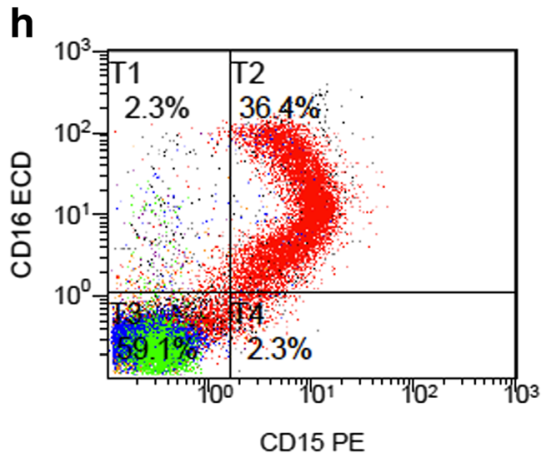

CD2 (g), dim CD13 (g), and dim to moderate CD64 (f) and was negative for CD10 (d), HLA-DR (e), CD34 (e), CD15 (h), and CD16 (h). The background granulocytic cells exhibited normal maturation, demonstrating the focal nature of APL in this patient $(\mathbf{h}-$ red population)

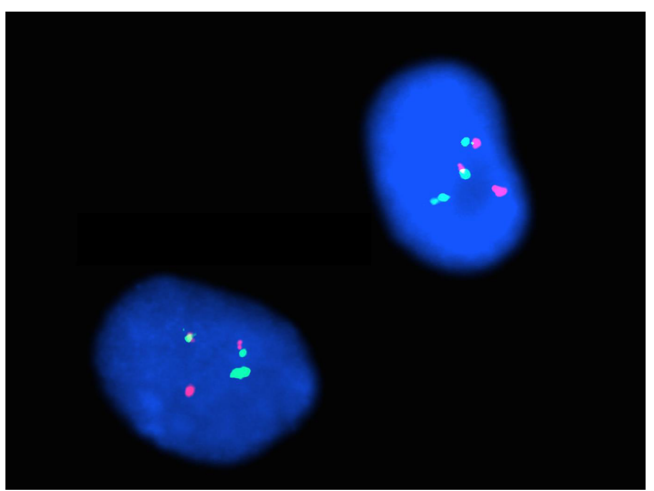

Fig. 4 FISH analysis. FISH analysis of the bone marrow demonstrated the $\mathrm{t}(15 ; 17)(\mathrm{q} 22 ; \mathrm{q} 12)$ PML/RARA translocation (fusion probe, red$15 \mathrm{q} 22$, green-17q12) 
case, the patient's leukemic cells were negative for CD56 and she responded well to chemotherapy.

\section{Conclusion}

Myeloid sarcoma as an initial presentation of acute promyelocytic leukemia is a very rare event. Patients with myeloid sarcoma should undergo bone marrow biopsies to rule out marrow involvement. Marrow involvement may be focal even at initial presentation; therefore, careful and thorough examination of the aspirate smear, clot section, and core biopsy is important in the workup of acute myeloid leukemia.

\section{Compliance with ethical standards}

Conflict of interest The authors declare that they have no conflict of interest.

\section{References}

1. Swerdlow SH, Campos E, Harris NL et al (2008) WHO classification of tumors of hematopoietic and lymphoid tissues, 4th edn. IARC Press, Lyon

2. Kyaw TZ, Maniam JAS, Bee PC et al (2012) Myeloid sarcoma: an unusual presentation of acute promyelocytic leukemia causing spinal cord compression. Turkish journal of hematology 29:278-282. doi:10.5505/tjh.2012.94809

3. Pacilli L, Lo Coco F, Ramadan SM et al (2010) Promyelocytic sarcoma of the spine: a case report and review of the literature. Advances in hematology. doi:10.1155/2010/137608

4. Campidelli C, Agostinelli C, Stitson R, Pileri SA (2009) Myeloid sarcoma: extramedullary manifestation of myeloid disorders. Am J Clin Pathol 132:426-337. doi:10.1309/AJCP1ZA7HYZKAZHS

5. Moulopoulos LA, Koutoulidis V (2015) Bone marrow MRI a pattern-based approach. Springer Milan, Milano. doi:10.1007/97888-470-5316-8

6. Brierley CK, Morgan EA, Sprague JR, Odejide OO, DeAngelo DJ, Steensma DP (2013) An extreme example of focal bone marrow involvement in acute myeloid leukemia. Am J Hematol 88:335336. doi:10.1002/ajh. 23350

7. Clark WB, Strickland SA, Barrett AJ, Savani BN (2010) Extramedullary relapses after allogeneic stem cell transplantation for acute myeloid leukemia and myelodysplastic syndrome. Haematologica 95:860-863. doi:10.3324/haematol.2010.025890 